\title{
Non-pharmacological interventions for depression/anxiety in older adults with physical comorbidities affecting functioning: systematic review and meta-analysis
}

\author{
Rachael Frost, (1) Yehudit Bauernfreund, and Kate Walters \\ Department of Primary Care and Population Health, University College London, London, UK
}

ABSTRACT

Objective: To review the effectiveness of non-pharmacological interventions in older adults with depression or anxiety and comorbidities affecting functioning.

Design: Systematic review and meta-analysis of randomized controlled trials, including searches of 10 databases (inception-Jul 2017).

Setting: Home/community.

Participants: People aged 60 and over experiencing functional difficulties from physical or cognitive comorbidities and have symptoms or a diagnosis of depression and/or anxiety.

Interventions: Non-pharmacological interventions targeted at depression/anxiety.

Measurements: We extracted outcome data on depressive symptoms, quality of life, functioning, and service use. We used random effects meta-analysis to pool study data where possible. Two authors assessed the risk of bias using the Cochrane Risk of Bias tool.

Results: We identified 14 eligible trials including 2099 randomized participants and two subgroup analyses. Problem-solving therapy (PST) reduced short-term clinician-rated depressive symptoms $(n=5$ trials, mean difference in Hamilton Depression Rating Scale score -4.94 [95\% CI -7.90 to -1.98]) but not remission, with limited evidence for effects on functioning and quality of life. There was limited high-quality evidence for other intervention types. Collaborative care did not appear to affect depressive symptoms, functioning, or quality of life; and had mixed evidence for effects upon remission. No intervention consistently affected service use, but trials were limited by small sample sizes and short follow-up periods. No anxiety interventions were identified.

Conclusion: PST may reduce depressive symptoms post-intervention in older people with depression and functional impairments. Collaborative care appears to have few effects in this population. Future research needs to assess cost-effectiveness, long-term outcomes, and anxiety interventions for this population.

Key words: depression, anxiety, meta-analysis, disability, medical comorbidity

\section{Introduction}

Late-life mental health is becoming an increasingly important issue. It is estimated that $37 \%-43 \%$ of older adults have symptoms of anxiety or depression (Braam et al., 2014; Rodda et al., 2011), while $9 \%-14 \%$ have a diagnosed anxiety or major depressive disorder (Rodda et al., 2011; Wolitzky-Taylor

Correspondence should be addressed to: Rachael Frost, Department of Primary Care and Population Health, University College London, Royal Free Campus, Rowland Hill Street, London NW3 2PF, UK. Phone: 0207830 2881. Email: rachael.frost@ucl.ac.uk. Received 23 Mar 2018; revision requested 29 Jun 2018; revised version received 09 Jul 2018; accepted 09 Aug 2018. First published online 27 November 2018. et al., 2010). Anxiety or depression in later life is associated with an increased risk of cognitive decline, functional decline, and increased use of healthcare services (Meeks et al., 2011; Wolitzky-Taylor et al., 2010). Frailer older adults, commonly experiencing physical or cognitive comorbidities affecting functioning (i.e. difficulties carrying out activities of daily living due to physical health conditions, in addition to depression), have a four-fold increase in the risk of clinically significant anxiety or depression (Ni Mhaolain et al., 2012). Comorbid physical and mental health disorders increases the risk of greater frailty, mortality, and primary and secondary healthcare service use 
(Djernes et al., 2011; Vaughan et al., 2015). In an ageing population, demand for mental health services specific to later life is likely to increase.

There is an abundance of evidence assessing the effectiveness of treatments for late-life depression. However, frailer older adults represent an understudied subgroup of this population, and there is evidence that physical illness, older age, and impaired executive functioning can negatively impact pharmacological and non-pharmacological treatment outcomes (Tunvirachaisakul et al., 2017). Antidepressants only appear to be effective when studied as a class, rather than individual drug types, and when reviews use an "older adult" threshold of $55+$ years rather than $65+$ (Jonsson et al., 2016; Kok et al., 2012). Antidepressant Randomized Controlled Trials (RCTs) do not account for frailty or malnutrition and the "older old $(75+$ years)" populations are underrepresented (Benraad et al., 2016), despite greater concerns about falls and polypharmacy in these populations. Collaborative care interventions and home care interventions have some evidence of effectiveness for depression, but the evidence base is very limited (Dham et al., 2017; Simning and Simons, 2017). Exercise interventions for depression have not been found to be effective in those with physical comorbidities (Schuch et al., 2016). Additionally, many older adults state a preference for psychological interventions (Gum et al., 2006; Mohlman, 2011).

However, secondary care services (e.g. community psychiatric services) focus mainly on severe mental illness, while center-based and intensive approaches may be unsuited to community-dwelling older adults with limited mobility and frailty. Cognitive behavioural therapy (CBT) shows smaller effect sizes for older rather than younger adults and has mostly been evaluated in samples with a mean age of 60-70 years old in people who are otherwise healthy (Gould et al. 2012a; 2012b). Existing psychological therapies offered in community settings may not fully account for issues such as increasing dependency, social isolation, reduced functional abilities and cognitive decline that are key for older people. However, some nonpharmacological therapies such as problem-solving therapy have shown promise in reducing disability, in addition to depression, in older adults with major depressive disorder and may offer promise for further research (Kirkham et al., 2016). Although some reviews have looked at frailer subgroup analyses of older adults (Jonsson et al., 2016), these have studied psychological therapies alone and documented "frailty indicators," rather than clearly defining an impaired population.

Consequently, the only review of this subpopulation with impairments as its main focus appears to have been carried out two decades previously (Landreville and Gervais, 1997). We, therefore, aimed to review the effectiveness of non-pharmacological interventions to reduce depression and anxiety in older adults with comorbidities affecting functioning.

\section{Methods}

We systematically reviewed Randomized Controlled Trials (RCTs) (Prospero registration CRD42017068441).

\section{Search strategy and inclusion criteria}

We developed a comprehensive search strategy (see Appendix A1) with terms based on age, impairments, depression/anxiety, and study type. We searched the following databases (inception-July 2017): MEDLINE, MEDLINE in Process \& Other Non-Indexed Citations, EMBASE, AMED, Web of Science: Social Science Citation Index, Cochrane Central Register of Controlled Trials and NHS Health Economic Evaluation Database, PsycINFO, Cumulative Index to Nursing and Allied Health Literature, Sociological Abstracts, Social Care Online, and Applied Social Sciences Index and Abstracts.

We performed additional searches of clinicaltrials.gov, UK Clinical Trials Gateway, and World Health Organization International Clinical Trials Registry Platform (inception-Sep 2017) to identify ongoing studies. We screened the reference lists of included studies/relevant systematic reviews and used forward citation tracking of all included studies. We used author searches to follow up conference abstracts, trials register entries, and protocols where available and necessary.

Our inclusion criteria were as follows:

- Participants: Older adults (aged $60+$ years); functional difficulties [including difficulties in activities of daily living (ADLs) or instrumental ADLs (IADLs), housebound, frail, low functioning scores on a validated scale, recipient of relevant support services (e.g. social care services)]; symptoms/diagnosis of depression and/or anxiety

- Interventions: Home- or community-based interventions delivered by any health, social, lay, or voluntary provider; single or multicomponent intervention aimed primarily at addressing depression or anxiety

- Comparator: Any

- Outcomes: Depressive and anxiety symptoms using validated questionnaires, other depression or anxiety outcomes (e.g. recovery), well-being, quality of life, functioning, service use

- Study type: Parallel-group, cluster, or crossover randomized controlled trials, economic evaluations of RCTs

We lowered our original age inclusion criterion from $75+$ to $60+$ years, as this allowed inclusion of 
a number of additional relevant studies of functionally impaired older adults with mean ages $60-75$.

We excluded interventions targeted at caregivers or targeted at specific health conditions (e.g. dementia, arthritis) in order to be more applicable to the wider frailer population, such as people described as generically "at risk" or "multimorbid" (unless there were documented associated difficulties in functioning); care-home interventions (recently reviewed by Simning and Simons [2017]); studies in which participants were not experiencing at least mild depression/anxiety; inpatient interventions; medication-only interventions (interventions containing a medication component were included); interventions focused primarily on another issue (e.g. frailty, falls prevention) in which depression/ anxiety is a secondary target as it would be difficult to be sure of the effective components; reviews, qualitative studies, quasi-experimental, and uncontrolled studies.

RF screened titles and abstracts, and YB independently checked $10 \%$ of these. We took an inclusive approach and screened the full texts of all studies assessing interventions in samples of depressed or anxious older adults, as we anticipated that difficulties in functioning may not be clearly reported in the abstract. RF and YB independently screened $10 \%$ of the full texts to ensure consistency in applying the inclusion criteria, with disagreements resolved through discussion, then each screened $50 \%$ of the remaining full texts. Dual review was undertaken for a further $7 \%$ of studies where there was uncertainty, with input from KW where needed. We contacted five authors for additional data to further inform about the inclusion criteria, and three replied. We sought further data regarding ten included studies from nine authors and eight replied, six of whom were able to provide further data.

\section{Data extraction and quality assessment}

We extracted data on participants, study type, intervention description (according to the TIDIER checklist [Hoffmann et al., 2014]), outcomes assessed, and main findings. If a study had multiple publications, we considered the main results paper as the primary paper and included information from related papers (e.g. protocols) where relevant. RF and YB independently assessed risk of bias using the Cochrane Risk of Bias tool (Higgins et al., 2011) and resolved disagreements through discussion. Overall ratings were judged on the least score counts; however, as nonpharmacological therapies are often compared to usual care and a placebo control is not always desirable or practical, we did not include participant blinding in the overall trial rating. Within "other bias," we assessed whether studies documented or controlled for use of antidepressants. Risk of bias ratings informed our narrative synthesis. We intended to assess publication bias using funnel plots but had insufficient data for this (Sterne et al., 2011).

\section{Synthesis}

We grouped studies according to intervention type: problem-solving therapy, other psychological therapies, and collaborative care (defined as complex interventions involving a multi-professional approach to care, a structured management plan, scheduled follow-ups, and enhanced interprofessional communication [Archer et al., 2012]). We conducted metaanalysis of similar outcomes post-intervention, the most relevant, and widely available timepoint (followup timepoint meta-analysis was precluded by differing outcome types and timepoints). Self-reported and clinician-rated outcomes were not combined as these produced different effect estimates in a previous meta-analysis (Cuijpers et al., 2010). We summarized effects for continuous data using mean difference (MD) or standardized mean difference (SMD), where appropriate, with weighting by inverse variance (Higgins and Green, 2011). For dichotomous data, we combined effects using odds ratios, weighted by the Mantel-Haenszel method (Higgins and Green, 2011). All analyses used a random effects model as we anticipated high clinical heterogeneity, quantified using the $\mathrm{I}^{2}$ statistic. Similar interventions within a single trial were aggregated into one intervention group for meta-analysis using Higgins and Green's (2011) formulae. Where necessary, we used $p$ values and test statistics to compute SDs, standardized mean difference, and $95 \%$ confidence intervals, then we combined studies using the generic inverse variance method (Higgins and Green, 2011).

RF performed meta-analysis using Revman 5.3 (The Nordic Cochrane Centre, The Cochrane Collaboration 2014). YB checked all data used in metaanalysis with the original papers or the authors' communications and independently verified calculations (e.g. calculation of standard deviations from $p$ values). We narratively summarized all other outcomes, including longer term follow-up timepoints.

\section{Results}

We identified 7708 unique references and screened 698 full texts (see Figure 1). Full texts were largely excluded due to their not targeting depression/ anxiety (in participants or intervention $[n=181]$ ), study type $(n=164)$, or not targeting an impaired older population $(n=154)$. We found five relevant ongoing trials as protocols, or trial register entries, 


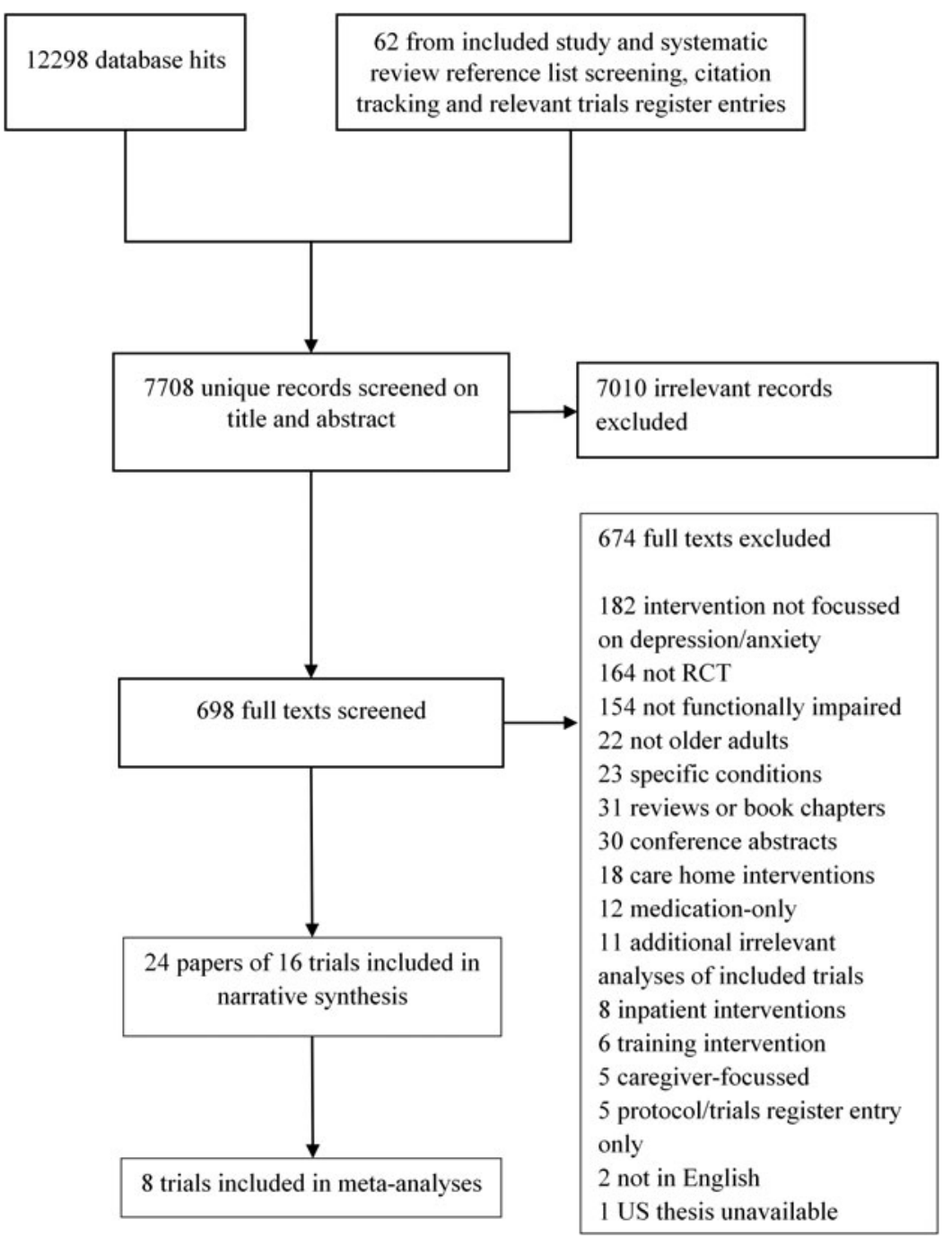

Figure 1. Flow diagram of studies throughout the review.

which had not previously been located (findings not currently reported, summarized in Appendix A3).

\section{Description of included studies}

We included 14 trials with a total of $n=2099$ randomized participants, comprising 11 RCTs (Alexopoulos et al., 2016; Banerjee et al., 1996; Choi et al., 2014; Ciechanowski et al., 2004; Ell et al., 2007; Enguidanos et al., 2005; Gellis et al., 2008; Kiosses et al., 2010; 2015; Nyunt 2010; Serrano et al., 2004), one cluster RCT (Bruce et al., 2015), one pilot RCT (Gellis et al., 2007), and one RCT with a non-concurrent control group (Llewellyn-Jones et al., 1999) (see Appendix A2 for a detailed summary of study characteristics). We also included two RCT subgroup analyses (Blanchard et al., 1995; Landreville and Bissonnette, 1997) (one $n$ not reported, one $n=23$ randomized). Most studies were carried out in the US $(n=10)$, with two UK studies (Banerjee et al., 1996; Blanchard et al., 1995), and one each in Australia (Llewellyn-Jones et al.,
1999), Singapore (Nyunt 2010), Spain (Serrano et al., 2004), and Canada (Landreville and Bissonnette, 1997). Sample sizes varied from 23 to 311.

Participant mean ages ranged from 64.8 to 84.9 years, with higher proportions of women in all trials (range from $69.6 \%$ to $87.5 \%$ ). Many studies captured a diverse population, including substantial proportions of ethnic minorities in studies reporting this data (see Table 1). Four studies focused on or included a considerable proportion of those with low incomes (Alexopoulos et al., 2016; Bruce et al., 2015; Choi et al., 2014; Landreville and Bissonnette, 1997) and a range of educational levels were reported. Many studies used a screening instrument to define depression criteria, with or without the Structured Clinical Interview for DSM-IV (SCID-IV) (see Table 1).

The majority of studies excluded people below a certain cognition threshold $(n=8)$ or those with dementia $(n=3)$ (see Table 1). Consequently, many studies, even if using a lower cognition threshold, reported mean baseline cognition scores within a 
Table 1. Characteristics of participants in each study

\begin{tabular}{|c|c|c|c|c|c|}
\hline STUDY ID & $\begin{array}{l}\text { DEPRESSION } \\
\text { SEVERITY } \\
\text { (CRITERIA) }\end{array}$ & $\begin{array}{l}\text { CRITERIA FOR } \\
\text { PHYSICAL } \\
\text { IMPAIRMENTS } \\
\text { AFFECTING } \\
\text { FUNCTIONING }\end{array}$ & $\begin{array}{c}\text { EDUCATION } \\
\text { (MEAN (SD) YEARS } \\
\text { OR \%LEVEL) }\end{array}$ & ETHNICITY & $\begin{array}{l}\text { COGNITION } \\
\text { INCLUSION } \\
\text { CRITERIA }\end{array}$ \\
\hline \multicolumn{6}{|l|}{ PST } \\
\hline $\begin{array}{l}\text { Alexopoulos } \\
2016\end{array}$ & $\begin{array}{l}\text { Major } \\
\quad(\text { SCID + SI })\end{array}$ & $\begin{array}{l}\text { Receiving support services, } \\
\text { IADL impairments }\end{array}$ & $13.2(2.9) \mathrm{yrs}$ & Not reported & $\begin{array}{l}\geq 24 \text { (MMSE) }+ \\
\text { no dementia diagnosis }\end{array}$ \\
\hline Choi 2014 & Major (SI) & $\begin{array}{l}\text { Homebound (Medicare } \\
\text { criteria), receiving } \\
\text { support services }\end{array}$ & $\begin{array}{l}<\text { high school } 7.6 \% \text {, high school } \\
19.6 \% \text {, some college } 33.5 \% \text {, } \\
\text { college degree } 13.9 \%, \\
\text { graduate school } 9.5 \%\end{array}$ & $\begin{array}{l}\text { Non-Hispanic White } 42.4 \% \text {, } \\
\text { African American/Black } 32.9 \% \text {, } \\
\text { Hispanic } 24.7 \%\end{array}$ & No dementia diagnosis \\
\hline $\begin{array}{l}\text { Ciechanowski } \\
2004\end{array}$ & Minor (SCID) & Receiving support services & Beyond high school $58 \%$ & $\begin{array}{l}\text { African American } 36 \% \text {, Asian } \\
\text { American } 4 \% \text {, Hispanic } 1 \% \text {, } \\
\text { American Indian } 1 \%\end{array}$ & $<3$ (6 item MMSE) \\
\hline Gellis 2007 & Major (SI) & Home care patients & 11.9 yrs (SD not reported) & $\begin{array}{l}\text { White } 80 \% \text {, Black } 10 \% \text {, } \\
\text { Hispanic } 10 \%\end{array}$ & $\geq 24$ (MMSE) \\
\hline Gellis 2008 & $\begin{array}{l}\text { Major } \\
\quad(\mathrm{SCID}+\mathrm{SI})\end{array}$ & Home care patients & $\begin{array}{l}\text { Int } 11.2 \text { yrs } \\
\text { Control } 11.34 \text { yrs } \\
\text { (SDs not reported) }\end{array}$ & $\begin{array}{l}\text { Int Caucasian 85\%, African } \\
\text { American 5\%, Hispanic 10\% } \\
\text { Control Caucasian 85\%, } \\
\text { African American 5\% } \\
\text { Hispanic } 10 \%\end{array}$ & $\geq 25$ (MMSE) \\
\hline Kiosses 2010 & $\begin{array}{l}\text { Major } \\
\quad(\mathrm{SCID}+\mathrm{SI})\end{array}$ & $\begin{array}{l}\text { IADL impairments, } \\
\text { mobility impairment }\end{array}$ & $\begin{array}{l}\text { Int } 12.50(3.67) \mathrm{yrs} \\
\text { Control } 12.23(2.68) \mathrm{yrs}\end{array}$ & $\begin{array}{l}\text { Caucasian } 73.33 \% \text {, African } \\
\text { American } 26.67 \%\end{array}$ & $\begin{array}{l}\geq 19 \text { (MMSE) plus } \\
\quad \leq 30 \text { (DRS IP) or } \\
\quad \leq 18 \text { (Stroop CW) }\end{array}$ \\
\hline \multirow[t]{2}{*}{ Kiosses 2015} & $\begin{array}{l}\text { Major } \\
\quad(\mathrm{SCID}+\mathrm{SI})\end{array}$ & $\begin{array}{l}\text { IADL impairments, } \\
\text { mobility impairment }\end{array}$ & Int 12.86 (3.37) yrs & $\begin{array}{l}\text { Int White } 81.08 \% \text {, African } \\
\text { American } 18.92 \% \text {, } \\
\text { Hispanic } 8.11 \%\end{array}$ & $\geq 17$ (MMSE) \\
\hline & & & Control 13.35 (2.72) yrs & $\begin{array}{l}\text { Control White } 83.78 \% \text {, } \\
\text { African American } \\
\text { 16.22\%, Hispanic } 0 \%\end{array}$ & \\
\hline \multicolumn{6}{|c|}{ Collaborative care } \\
\hline Banerjee 1996 & Major (SI) & Home care patients & Not reported & Not reported & Not reported \\
\hline Blanchard 1995 & Mixed (SI) & Mobility impairment & Not reported & Not reported & Not reported \\
\hline Bruce 2015 & Mixed (SI) & Home care patients & $12.0(3.5) \mathrm{yrs}$ & $\begin{array}{l}\text { White } 80.7 \% \text {, Black } 18.0 \% \text {, } \\
\text { Other } 1.3 \%\end{array}$ & No dementia diagnosis \\
\hline Ell 2007 & Mixed (SI) & Home care patients & Not reported & $\begin{array}{l}\text { Int Non-Hispanic White } \\
75 \% \text {, Control Non-Hispanic } \\
\text { White } 69 \%\end{array}$ & $\begin{array}{l}\text { Cognitive impairment } \\
\text { precluding consent }\end{array}$ \\
\hline
\end{tabular}




\begin{tabular}{|c|c|c|c|c|c|}
\hline STUDY ID & $\begin{array}{l}\text { DEPRESSION } \\
\text { SEVERITY } \\
\text { (CRITERIA) }\end{array}$ & $\begin{array}{c}\text { CRITERIA FOR } \\
\text { PHYSICAL } \\
\text { IMPAIRMENTS } \\
\text { AFFECTING } \\
\text { FUNCTIONING }\end{array}$ & $\begin{array}{l}\text { EDUCATION } \\
\text { (MEAN (SD) YEARS } \\
\text { OR \%LEVEL) }\end{array}$ & ETHNICITY & $\begin{array}{l}\text { COGNITION } \\
\text { INCLUSION } \\
\text { CRITERIA }\end{array}$ \\
\hline Enguidanos 2005 & Mixed (SI) & ADL impairments & $\begin{array}{l}<\text { high school } 40 \% \text {, high school } \\
33 \% \text {, some college } 27 \%\end{array}$ & $\begin{array}{l}\text { White } 66 \% \text {, African American } 17 \% \text {, } \\
\text { Latino } 13 \% \text {, Asian } 4 \% \text {, Native } \\
\text { American } 1.3 \%\end{array}$ & $\leq 4$ (SPMSQ) \\
\hline $\begin{array}{l}\text { Llewellyn-Jones } \\
1999\end{array}$ & Mixed (SI) & $\begin{array}{l}\text { Residing in supported } \\
\text { living }\end{array}$ & Not reported & Not reported & $\geq 18$ (MMSE) \\
\hline Nyunt 2010 & Minor (SI) & $\begin{array}{l}\text { Receiving support } \\
\text { services, residing } \\
\text { in supported living }\end{array}$ & $\begin{array}{l}50.9 \%(\mathrm{UC}) \text { and } 46.1 \% \\
\text { (CC) were illiterate }\end{array}$ & $\begin{array}{l}\text { Int Chinese } 84.3 \% \\
\text { Control Chinese } 85.7 \%\end{array}$ & No dementia diagnosis \\
\hline $\begin{array}{l}\text { Bibliotherapy } \\
\text { Landreville } 1997\end{array}$ & Mixed (SI) & $\begin{array}{l}\text { ADL or IADL impairments, } \\
\text { mobility impairment }\end{array}$ & $\begin{array}{l}\text { Int } 7.80 \text { yrs } \\
\text { Control } 8.53 \text { yrs } \\
\text { (SDs not reported) }\end{array}$ & Not reported & Not reported \\
\hline \multicolumn{6}{|c|}{ Life review therapy } \\
\hline Serrano 2004 & Mixed (SI) & Receiving support services & $\begin{array}{l}\text { Literate } 7 \% \text {, elementary school } \\
67.4 \% \text {, secondary school } \\
23.3 \% \text {, university } 2.3 \%\end{array}$ & Not reported & $\geq 28$ (MMSE) \\
\hline
\end{tabular}

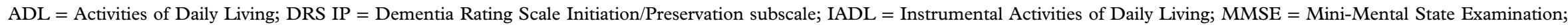

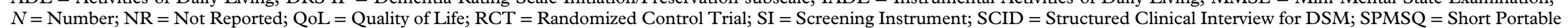
Mental Status Questionnaire; Stroop CW $=$ Stroop Color-Word test. 
normal range (i.e. Mini-Mental State Examination (MMSE) scores between 26.8 and 29.3 across studies (Alexopoulos et al., 2016; Bruce et al., 2015; Llewellyn-Jones et al., 1999), 1.58 on the Short Portable Mental Status Questionnaire (Enguidanos et al., 2005), and 5.5 on the 6-item MMSE [Ciechanowski et al., 2004]). Only two studies included people with greater cognitive impairment (Kiosses et al., 2015) or executive dysfunction (Kiosses et al., 2010) resulting in a wider range of cognition scores, but still normal mean cognitive function in Kiosses et al (2010). Two studies did not report any cognition data (Banerjee et al., 1996; Blanchard et al., 1995).

\section{Risk of bias in included studies}

Overall, most studies were at an unclear risk of bias (see Figure 2), largely due to limited reporting of random sequence generation $(n=8)$, allocation concealment $(n=13)$, and lack of an online or published protocol to assess selective reporting $(n=11)$. Studies were most commonly at a low risk of bias for outcome assessment $(n=12)$, incomplete outcome data $(n=10)$, and reporting or controlling for antidepressant use (other bias, $n=9$ ). Studies were most commonly at a high risk of bias for participant blinding $(n=7)$, although this was expected as blinding participants can be difficult in trials of complex interventions and so these ratings were not included in the overall score. Individual risk of bias ratings are discussed throughout the synthesis for each outcome.

\section{Modified problem-solving therapy ( $n=7$ trials, including $n=688$ randomized participants)}

Problem-solving therapy (PST) aims to systematically identify and address daily life problems to reduce depression and improve future coping skills (Ciechanowski et al., 2004) through standard steps including: identifying problems, establishing achievable goals, brainstorming solutions, using decisionmaking guidelines, evaluating and contrasting solutions, developing action plans, and evaluating outcomes. PST was modified in some studies to suit a more impaired population:

- Integration into existing home care services, with six sessions to fit home care's fast pace and limited resources and individuals' frailty (Gellis et al., 2007; 2008)

- Emphasizing social activation, increasing outdoor interactions, and developing an exercise program (Ciechanowski et al., 2004)

- Problem Adaptation Therapy with environmental adaptations to circumvent behavioral and/or functional limitations and involving willing/available caregivers over 12 weeks (Kiosses et al., 2010; 2015)

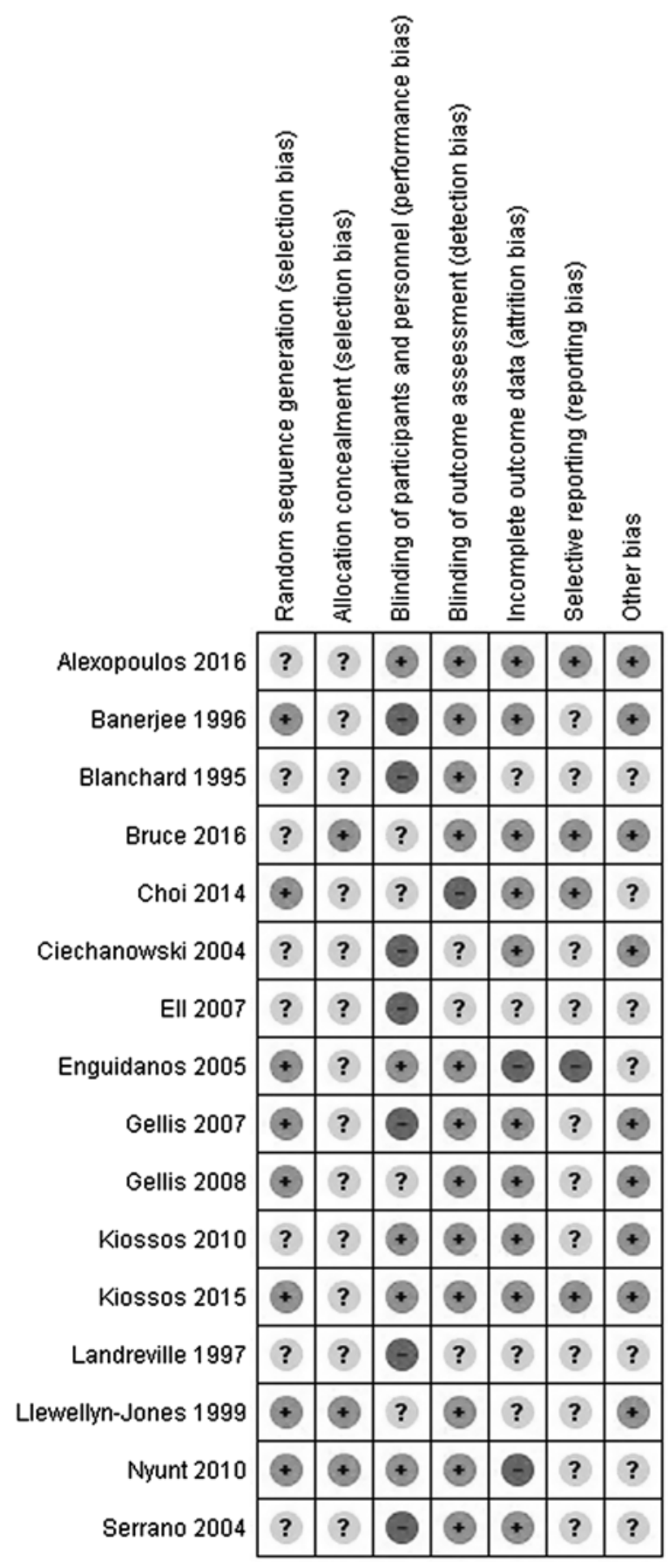

Figure 2. Risk of bias in included studies (NB selective reporting judgment refers to depressive symptoms and is highlighted where different for other outcomes in the text).

- Delivery over videoconferencing software (telePST) to enable home-based therapy (Choi et al., 2014)

- Increasing pleasant events (Choi et al., 2014; Ciechanowski et al., 2004; Gellis et al., 2007; 2008; Kiosses et al., 2010)

PST was most commonly delivered by social workers (usually Masters-level) (Alexopoulos et al., 2016; Choi et al., 2014; Ciechanowski et al., 2004; 
Gellis et al., 2007; Kiosses et al., 2010), registered nurses (Ciechanowski et al., 2004) or clinical psychologists, and clinical doctorate candidates (Kiosses et al., 2015). All delivered PST faceto-face at participants' homes over 6-12 sessions, apart from one trial comparing delivery over Skype to face-to-face and to-care calls (Choi et al., 2014) (collapsed to a single PST group for meta-analysis as per Higgins and Green [2011]). Other trial comparators included usual care (Ciechanowski et al., 2004), enhanced usual care (e.g. with basic education) (Gellis et al., 2007; 2008) or attention controls (e.g. supportive therapy) (Kiosses et al., 2010; 2015). One compared case management plus PST to case management alone (Alexopoulos et al., 2016).

\section{DePRESSIVE SYMPTOMS}

Modified PST significantly reduced clinicianrated Hamilton Depression Rating Scale (HAM-D) scores post-intervention (see Figure 3[a], $n=5, \mathrm{MD}$ -4.94 [95\% CI -7.90 to -1.98 ], $\mathrm{p}=0.001$ ). Two further studies found significantly lower scores on other measures, including the Montgomery-Åsberg Depression Rating Scale (MADRS), compared to supportive therapy (Kiosses et al., 2015) and 20item Hopkins Symptom Checklist Depression Scale (HCSL-20) compared to usual care (Ciechanowski et al., 2004). Reduced depressive symptoms were maintained 6-12 months from baseline in studies assessing this outcome (Alexopoulos et al., 2016; Choi et al., 2014; Ciechanowski et al., 2004; Gellis et al., 2008). All studies were at an overall unclear risk of bias (with some low-risk domains), apart from Choi et al. (2014), who used non-blinded outcome assessors. However, PST did not significantly increase the post-intervention clinician-rated odds of response ( $\geq 50 \%$ symptom reduction from baseline, OR 3.24 [ $95 \%$ CI 0.52 to 20.27], Figure 3[b]) or remission (OR 2.62 [95\%CI 0.57 to 12.16 ], Figure 3[c]) using the HAM-D (Alexopoulos et al., 2016), HSCL-20 (Ciechanowski et al., 2004), and MADRS (Kiosses et al., 2015). Similarly, no effects were found 12 weeks post-intervention (Alexopoulos et al., 2016) or at the 36-week (Choi et al., 2014) or 12-month follow-up (Ciechanowski et al., 2004). Kiosses et al. (2015) did not assess longer term outcomes.

We could not meta-analyze self-reported depressive symptoms, as two papers reported identical mean and SD 15-item Geriatric Depression Scale (GDS-15) scores at all timepoints, suggesting a reporting error (Gellis et al., 2007; 2008). These figures did however, indicate significantly lower symptoms at post-intervention, 3 months, and 6 months (Gellis et al., 2007; 2008). One also found significantly lower Beck Depression Inventory scores post-intervention (PST vs. usual care, 10.20 vs $27.4, \mathrm{p}<0.001$ ), maintained at 6 months (Gellis et al., 2007).
Functioning AND Disability

PST did not significantly reduce self-reported disability post-intervention (Figure $3[\mathrm{~d}], n=4$, SMD -1.35 [95\% CI -2.96 to 0.25 ], $\mathrm{p}=0.10$ ) when assessed using the World Health Organization Disability Assessment Schedule and the Sheehan Disability Scale. Within one study, significant effects upon disability were maintained at 24 weeks for tele-PST and face-to-face PST and at 36 weeks for tele-PST (Choi et al., 2014). Another study, similarly to post-intervention, found no effects at the 12-week follow-up (Alexopoulos et al., 2016).

\section{QuAlity OF LIFE}

Evidence about the impact of PST upon the quality of life was mixed across two studies using the Quality of Life Inventory. Meta-analysis was not undertaken as the SDs (but not means) reported were identical in both papers at all timepoints (Gellis et al., 2007; 2008), suggesting a potential reporting error. Significantly higher quality of life was found postintervention and at 3 and 6 months in Gellis et al. (2007) but no effects were found in Gellis et al. (2008). Within Ciechanowski et al. (2004) there were improvements in emotional and functional but not social or physical well-being over 12 months on the Functional Assessment of Cancer Therapy Scale: General.

Service use and costs

None of the three studies reporting service use found PST to significantly affect primary, secondary, or home care service use over the treatment period or longer follow up, although this was considered beneficial where PST was delivered as part of home care (Ciechanowski et al., 2004; Gellis et al., $2007 ; 2008)$. One study reported intervention costs (\$630 per patient) (Ciechanowski et al., 2004), and two noted that social work home visits were reimbursable by Medicare (Gellis et al., 2007; 2008). No other studies reported this data.

\section{Collaborative care interventions ( $n=7$ studies including $n=1351$ participants)}

Seven studies evaluated collaborative care interventions, provided in the community or in care services (components listed in Table 2). Key intervention providers were usually nurses (Blanchard et al., 1995; Bruce et al., 2015; Ell et al., 2007; Nyunt 2010), but could include social workers, psychologists (Ell et al., 2007), or medical staff (Banerjee et al., 1996).

Baseline depressive symptoms levels were mainly mixed (Blanchard et al., 1995; Bruce et al., 2015; Ell et al., 2007; Enguidanos et al., 2005), with two studies focusing on major depression 
(a)

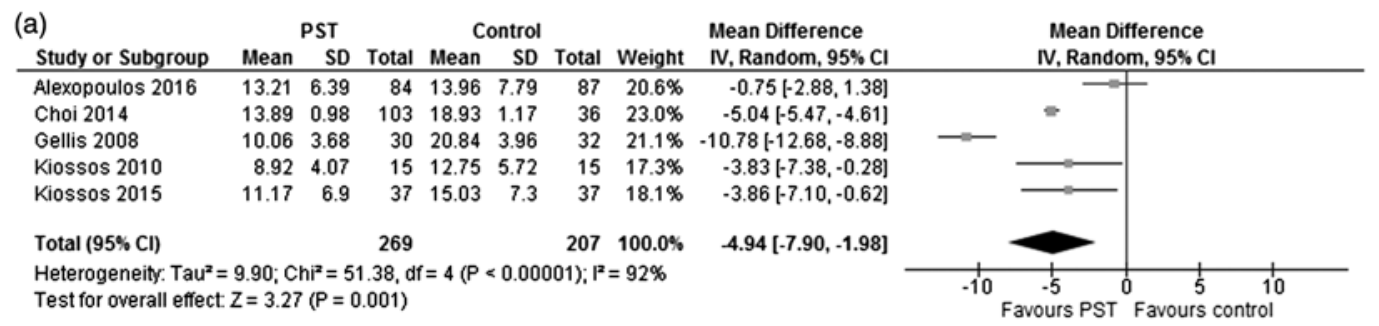

(b)

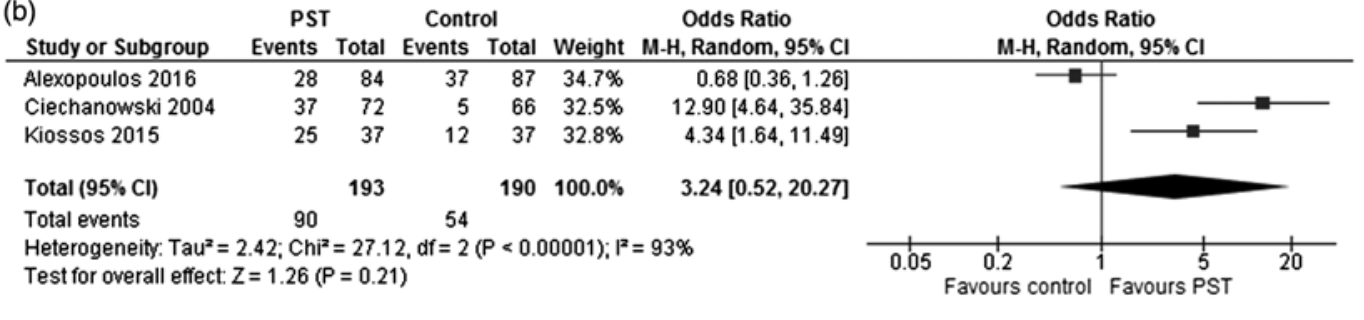

(c)

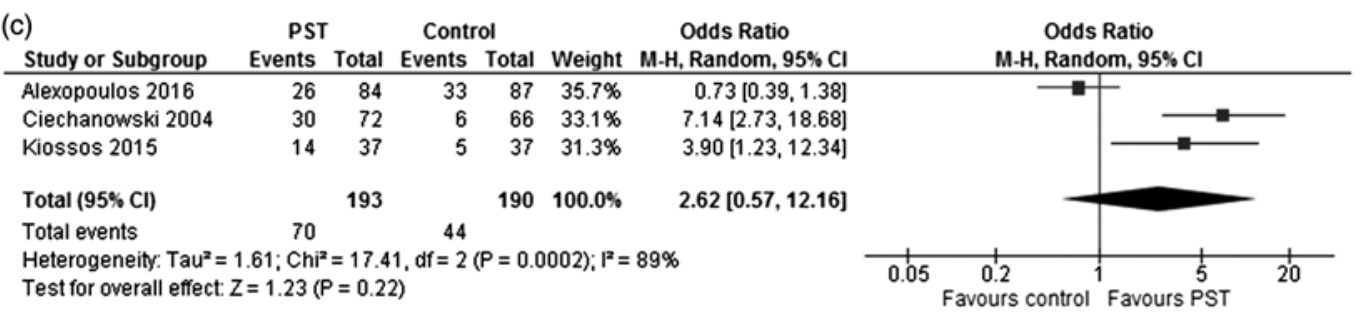

(d)

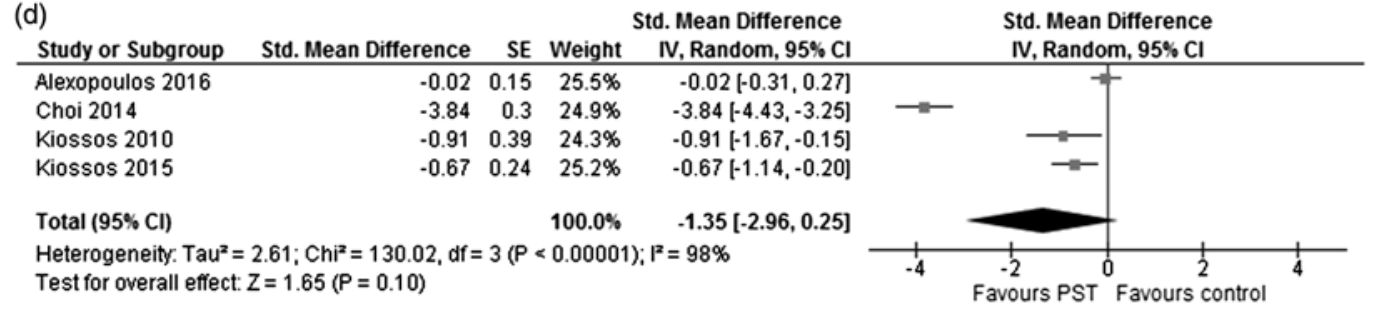

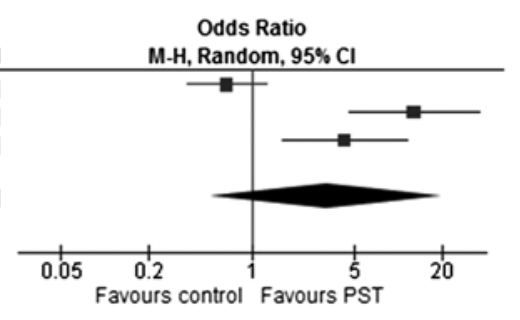

Odds Ratio

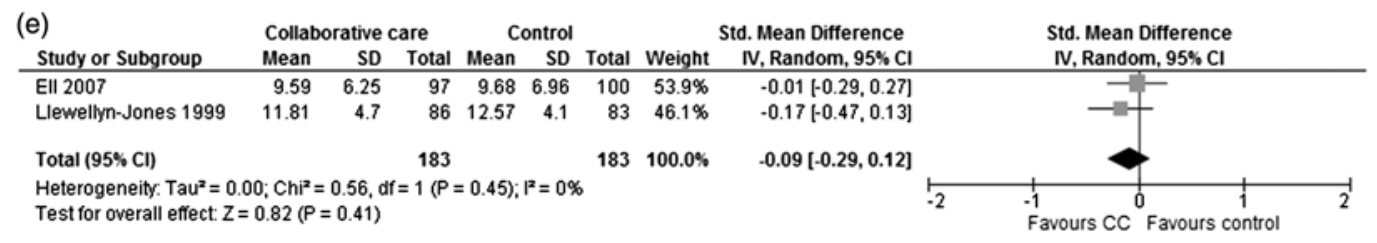

Figure 3. Forest plots of meta-analyses: (a) meta-analysis of the effectiveness of problem-solving therapy upon clinician-rated depressive symptoms; (b) meta-analysis of the effectiveness of problem-solving therapy upon response ( $\geq 50 \%$ reduction in symptoms from baseline); (c) meta-analysis of the effectiveness of problem-solving therapy upon remission (not depressed according to threshold); (d) meta-analysis of the effectiveness of problem-solving therapy upon self-reported disability; and (e) meta-analysis of the effects of collaborative care upon self-reported depressive symptoms.

(Banerjee et al., 1996; Llewellyn-Jones et al., 1999) and one on minor depression (Nyunt 2010). Comparators included "routine care" (further details not reported) (Llewellyn-Jones et al., 1999); GP management with or without notification of depression severity (Banerjee et al., 1996; Blanchard et al., 1995; Ell et al., 2007; Nyunt 2010); usual geriatric case management with care plan (Enguidanos et al., 2005); and usual care enhanced by screening, notifying GPs (Ell et al., 2007), or following agency procedures (Bruce et al., 2015) if the patient did not improve.
Potential meta-analysis for collaborative care interventions relied mainly on data from an unpublished thesis, Nyunt (2010), whose analysis was at high risk of bias as they did not use an intentionto-treat analysis. We, therefore, only undertook meta-analysis where higher quality data were available (self-reported depressive symptoms).

EFFECTS UPON DEPRESSive SyMptoms $(N=7)$ There were mixed effects on continuous depressive symptom scores. Two large studies found no effects on clinician-rated HAM-D scores post-intervention 
Table 2. Collaborative care intervention content

\begin{tabular}{|c|c|c|c|c|c|c|c|c|c|c|c|}
\hline \multirow[b]{3}{*}{ STUDY } & \multirow[b]{3}{*}{ LOCATION } & \multicolumn{10}{|c|}{ INTERVENTION COMPONENT } \\
\hline & & \multicolumn{5}{|c|}{ STAFF } & \multicolumn{5}{|c|}{ PATIENT } \\
\hline & & $\begin{array}{c}\text { SPECIFIC } \\
\text { DEPRESSION } \\
\text { CASE } \\
\text { MANAGER } \\
\text { IDENTIFIED }\end{array}$ & MONITORING & $\begin{array}{c}\text { MDT } \\
\text { TEAM } \\
\text { MEETINGS }\end{array}$ & $\begin{array}{c}\text { PROVIDER } \\
\text { EDUCATION }\end{array}$ & $\begin{array}{c}\text { ENHANCED } \\
\text { COORDINATION/ } \\
\text { COMMUNICATION } \\
\text { ETWEEN } \\
\text { PROVIDERS }\end{array}$ & $\begin{array}{l}\text { MDT } \\
\text { MANAGEMENT } \\
\text { PLANS }\end{array}$ & $\begin{array}{c}\text { PATIENT } \\
\text { EDUCATION }\end{array}$ & $\begin{array}{l}\text { PSYCHOLOGICAL } \\
\text { THERAPY } \\
\text { OFFERED }\end{array}$ & $\begin{array}{c}\text { PRESCRIPTION/ } \\
\text { MANAGEMENT } \\
\text { OF } \\
\text { ANTIDE- } \\
\text { PRESSANTS }\end{array}$ & $\begin{array}{l}\text { OTHER } \\
\text { (E.G. SOCIAL } \\
\text { ACTIVITIES } \\
\text { OFFERED) }\end{array}$ \\
\hline Banerjee $1^{1996}{ }^{29}$ & Community & $\checkmark$ & $\checkmark$ & $\checkmark$ & & $\checkmark$ & $\checkmark$ & & $\checkmark$ & $\checkmark$ & $\checkmark$ \\
\hline Blanchard $1995^{39}$ & Community & $\checkmark$ & $\checkmark$ & $\checkmark$ & & $\checkmark$ & $\checkmark$ & & $\checkmark$ & $\checkmark$ & $\checkmark$ \\
\hline Bruce $2015^{36}$ & $\begin{array}{l}\text { Home care } \\
\text { services }\end{array}$ & & $\checkmark$ & & $\checkmark$ & $\checkmark$ & & $\checkmark$ & $\begin{array}{c}\checkmark \\
\text { (goal } \\
\text { setting } \\
\text { only) }\end{array}$ & $\checkmark$ & \\
\hline Ell $2007^{28}$ & $\begin{array}{l}\text { Home care } \\
\text { services }\end{array}$ & $\checkmark$ & $\checkmark$ & & $\checkmark$ & $\checkmark$ & & & $\checkmark$ & $\checkmark$ & \\
\hline $\begin{array}{l}\text { Enguidanos } \\
\mathbf{2 0 0 5}^{30}\end{array}$ & $\begin{array}{l}\text { Geriatric care } \\
\text { management } \\
\text { services }\end{array}$ & & $\checkmark$ & $\checkmark$ & & $\checkmark$ & & & $\checkmark$ & $\checkmark$ & \\
\hline $\begin{array}{l}\text { Llewellyn- } \\
\text { Jones } \\
\text { 1999 }^{38}\end{array}$ & $\begin{array}{l}\text { Assisted living } \\
\text { and hostel } \\
\text { facilities }\end{array}$ & & $\checkmark$ & $\checkmark$ & $\checkmark$ & $\checkmark$ & & $\checkmark$ & $\checkmark$ & $\checkmark$ & $\checkmark$ \\
\hline Nyunt $2010^{33}$ & Community & $\checkmark$ & $\checkmark$ & & $\checkmark$ & $\checkmark$ & & $\checkmark$ & $\checkmark$ & $\checkmark$ & \\
\hline
\end{tabular}

*aka key worker, case worker, Clinical Depression Specialist etc.

MDT $=$ Multidisciplinary Team. 
or at 12 months (Bruce et al., 2015; Nyunt 2010). A subgroup with major depression and one smaller study $(n=69)$ found significant reductions in depressive symptoms (Banerjee et al., 1996; Bruce et al., 2015), while Blanchard et al.'s (1995) subgroup analysis found greater symptom improvements in those with physical capacity than those without, although this did not interact with the treatment group. There were similarly no effects upon post-intervention self-reported depressive symptoms $(n=2$, SMD -0.09 [95\%CI -0.29 to $0.12], p=0.41$, Figure $3[\mathrm{e}])$. A further unpublished study (at high risk of bias due to higher intervention arm dropout rates) found no differences in 9-item Patient Health Questionnaire (PHQ-9) scores postintervention or at 12 months (Enguidanos et al., 2005). Nyunt (2010) (at high risk of bias) found some differences in GDS and BDI scores postintervention but not at 12 months.

Odds of depression remission were mixed. Banerjee et al. (1996) found significantly higher odds of clinician-rated remission using Geriatric Mental State AGECAT categories, while Nyunt (2010) found no differences in remission using HAM-D at any timepoint. Odds of self-reported remission was significantly higher using the GDS-15 (Nyunt 2010) with significant positive movement into "less depressed" categories using the GDS-30 cutoffs (Llewellyn-Jones et al., 1999); but not using the BDI (Nyunt 2010) or in the odds of response using the PHQ-9 at 4, 8, or 12 months (Ell et al., 2007).

Functioning $(N=2)$

There was no evidence that collaborative care affected 12-item Short Form (SF-12) physical functioning subscale scores, Mahoney and Barthel Activities of Daily Living (ADL) scores, Lawton Instrumental Activities of Daily Living (IADL) scores (Nyunt 2010), or SF-20 scores (Ell et al., 2007) at any timepoint up to 12 months.

Quality of Life $(N=2)$

Mental functioning scores (SF-12) were significantly higher post-intervention in the collaborative care group compared to usual care but not at 12 months in Nyunt (2010), and Ell et al. (2007) found no differences in odds of an improvement in mental, social, or role functioning scores.

Service use ANd Costs $(N=5)$

One study found significant effects for collaborative care on the number of days in hospital (3.59 vs. $6.31, \mathrm{p}=0.04$, unpublished data supplied by authors) (Enguidanos et al., 2005). However, one large cluster trial found no significant reductions in risk of 30- or 60-day hospitalization from home health services (except when including service-level data from all people in all clusters, rather than those agreeing to full-study participation) (Bruce et al., 2016). Others similarly found no differences in the 12-month odds of hospitalization (meta-analysis not possible due to insufficient data) (Ell et al., 2007; Nyunt 2010), home care visits (Ell et al., 2007; Enguidanos et al., 2005), primary care or physician visits (Enguidanos et al., 2005; Nyunt 2010), home care readmissions (Ell et al., 2007), moves to long-term care (Enguidanos et al., 2005), or emergency department visits (Ell et al., 2007; Enguidanos et al., 2005; Llewellyn-Jones et al., 1999). Only Enguidanos et al. (2005) calculated healthcare costs (unpublished), which were significantly lower at four months (\$3295 vs. $\$ 5417$, $\mathrm{p}=0.04)$ and 12 months ( $\$ 8403$ vs. $\$ 11,242$, $\mathrm{p}=0.05)$. Banerjee et al. (1996) reported extra costs of a part time doctor as a depression case manager but did not cost this.

\section{OTHER PSYCHOLOGICAL THERAPIES}

The evidence base available for other psychological therapies in this population consisted of small single studies at an unclear risk of bias. Four sessions of life review therapy that focused on different life periods had significant post-intervention effects on selfreported depressive symptoms (20-item Center for Epidemiological Studies-Depression Scale) and life satisfaction (Life Satisfaction Index A), compared to social visits in 50 social care services clients (Serrano et al., 2004). Bibliotherapy (i.e. reading a CBT-based book Feeling Good over 4 weeks) reduced depressive symptoms in two out of three scales (BDI, GDS-30, Inventory to Diagnose Depression) compared to weekly supportive phone calls in a subgroup analysis of 23 people with disability in at $\geq 1 \mathrm{ADL}$, IADL or mobility, with no effect upon functioning (Landreville and Bissonnette, 1997). Neither study reported service use or cost data.

\section{Discussion}

Within this review, we included 14 RCTs including 2099 randomized individuals, plus two subgroup analyses, primarily assessing modified problemsolving therapy $(n=7)$ and collaborative care interventions $(n=7)$. For older adults with depression and physical comorbidities affecting functioning, we found that home-based PST significantly reduced depressive symptoms, but did not affect remission, functioning, or service use, and showed mixed effects on quality of life. The evidence for collaborative care was heterogeneous, with no effects on depressive symptoms in meta-analysis and narrative 
synthesis showed little effect upon quality of life, service use or functioning, and mixed effects upon remission. The evidence base for bibliotherapy and life review was too limited to draw conclusions. No treatments for anxiety were identified in this population.

Similar effect sizes for PST on depressive symptoms have been noted in the general older adult population (Jonsson et al., 2016; Kirkham et al., 2016). However, Kirkham et al. (2016) also found effects on disability. We included a greater number of trials than Kirkham et al, and outcomes appeared to be broadly consistent between different measures used. It is, therefore, likely that either trials were underpowered to detect differences (as functioning was never a primary outcome), or the results were mainly affected by a single study in which both study arms also received case management (Alexopoulos et al., 2016). The latter study may also be the reason for the lack of effect on remission despite a strong effect on symptoms or this may be due to the high baseline depressive symptom scores (e.g. HAM-D scores of 21-24). Heterogeneity was also very high $(\geq 90 \%)$ for some PST outcomes, which could relate to differences in timepoints (e.g. immediately postintervention or some weeks after) or intervention components. There were too few studies reporting quality of life outcomes to draw firm conclusions, and quality of life appears to get little attention in trials assessing late-life depression treatments (Jonsson et al., 2016; Kirkham et al., 2016). While a few larger studies have shown positive effects upon late-life depression for primary care-based collaborative care in the general older adult populations, smaller studies in other settings indicate mixed effects (Dham et al., 2017) similarly to our review. However, an individual patient meta-analysis has reported similar collaborative care effects regardless of the number or type of chronic physical conditions across all ages (Panagioti et al., 2016).

Although executive dysfunction has been linked to worse treatment outcomes (Tunvirachaisakul et al., 2017), the two studies including participants with a wider range of cognition scores (Kiosses et al., 2010; 2015) found results consistent with those restricted to normal scores, suggesting that PST may be equally effective in a cognitively impaired population. Mixed reporting of cognition and mixed results in collaborative care studies mean that conclusions cannot be drawn for these interventions.

\section{Strengths and limitations of the evidence base}

The evidence base was largely at an unclear risk of bias due to poor reporting. Studies at high risk of bias were rare, apart from a few key collaborative care trials. Regarding generalizability, the included studies recruited ethnically and socioeconomically diverse participants; however, most were carried out in the US and all had a higher proportion of female participants. Although within most studies participants fell within a "normal" cognition range, some studies included people with a range of cognition levels that included those with cognitive impairment. Within this review, PST appears to have consistent effects on depression in studies with participants who have greater cognitive impairment (Kiosses et al., 2010; 2015) and those with executive dysfunction but no physical disability (Alexopoulos et al., 2003; Areán et al., 2010).

Post-intervention effectiveness was well-documented, but follow-up periods of over one year were rare. Previous research suggests that depression is associated with faster physical decline and greater health service use (Djernes et al., 2011; Vaughan et al., 2015). Reducing depression may maintain physical functioning, rather than improve it, which may only be apparent in larger samples over longer follow up. Quality of life information was limited, while adverse event data were omitted by all but two studies (Banerjee et al., 1996; Kiosses et al., 2015). We found no trials of CBT or any therapies for anxiety targeted to our population. Attrition in many studies was similar across control and treatment arms, suggesting that dropout was not an issue likely to affect review results. However, collaborative care studies had mixed fidelity and adherence in those measuring it, which may be a source of heterogeneity in outcomes (e.g. in Ell et al. [2007] 30\% received no intervention care). Within PST, generally high fidelity scores were reported and where assessed, participant attendance was fairly high, even for studies with greater numbers of sessions. Book-based CBT had very low adherence (on average only half of the book was read [Landreville and Bissonnette, 1997]).

\section{Strengths and limitations of the review}

We searched a wide range of databases using comprehensive search terms, and additional methods located only one extra subgroup analysis and one small study. One reviewer assessed titles, abstracts, and full texts. However, they took an inclusive approach and a proportion were screened independently by a second reviewer with good agreement. We did not include trials of interventions for depression in older people as a general population. Although these may include some participants with difficulties in functioning arising from physical comorbidities, they are only likely to be a small proportion as the 
mean age within many of these trials is $65-70$ (Gould et al., 2012b; 2012a). However, we took an inclusive approach to screening to identify larger trials that included a subgroup analysis in a frailer population. Two independent reviewers assessed risk of bias and extracted meta-analysis data. Meta-analysis was limited by inconsistencies in outcome timepoints (e.g. immediately post-intervention to six weeks after), differing intervention lengths and poor study quality. We received a good response from authors to our requests for further data.

\section{Implications for practice}

Currently, UK psychological therapy services commonly focus primarily on delivering CBT for depression and anxiety, with low referral to and uptake of these services in older people (Walters et al., 2018; Wang et al., 2005). In our review, we found no evidence regarding the use of CBT in older people with physical comorbidities affecting functioning. It may be that CBT is more cognitively demanding than other therapies and so has not been trialed in those who are frailer and more likely to have mild cognitive impairment. Although there is some evidence for its effectiveness in the general older adult population, effect sizes are smaller than for younger people (Gould et al., 2012a; 2012b). Also, frailer people may find CBT difficult or even detrimental to apply when their problems or negative thoughts may be realistic and valid (Isaacowitz and Seligman, 2002).

Home-based problem-solving therapy, delivered by non-mental health specialists, in perhaps as few as six sessions, may be effective in this population with moderate-severe depressive symptoms, at least shortterm and should be considered in practice. The effect size is slightly higher for modified PST (1.79 [3.39, 0.20 ], converting the $M D$ from Figure 3[a] to SMD) than that reported for SSRIs in general older adult populations (SMD 1.2 [0.3-2.1]) (Kok et al., 2012). PST also has a high level of acceptability (Gellis et al., 2007; 2008; Kiosses et al., 2010; 2015) and may address the importance placed by older people with complex problems upon feeling a sense of mastery about solving issues and helping a combination of issues that older people with complex problems attribute depression to (Von Faber et al., 2016). Provision of this alternative therapy in routine care may support increasing the access to mental health support for frail older people. The evidence for collaborative care is more conflicting and insufficient to make recommendations. Bibliotherapy and 'life review' (a therapy including the recall, evaluation and integration of life experiences to achieve a positive sense of self in the later stages of life) (Lan et al., 2017) lacked sufficient evidence to make recommendations for this group.

\section{Implications for research}

PST showed positive short-term effects for reducing depressive symptoms, although it did not show effects upon other outcomes. Large scale RCTs, therefore, need to be powered to assess the effects of PST upon a more comprehensive set of outcomes more relevant to older people, particularly functioning (a key frailty outcome) (Ferrucci et al., 2004) and quality of life (Lenze et al., 2016), especially in the long term. Evaluation of hospitalizations, social care use, and cost-effectiveness is also vital to assist commissioning decisions, as home-based services are potentially costly but may deliver long-term costsavings in health or social care use. Other nonpharmacological interventions for depression that have shown to be effective in the general older adult populations, such as CBT, behavioral activation, and life review (Gould et al., 2012a; 2012b; Lan et al., 2017; Orgeta et al., 2017), may also offer promise, but could be optimized through working with frail older people to understand how these treatments could be better tailored to their needs and abilities. Book-based CBT had low adherence in this population and exercise interventions do not appear to be effective when people have a medical comorbidity (Schuch et al., 2016), so these may be less useful avenues for investigation. Research into interventions for anxiety (and comorbid anxiety and depression, which negatively impact upon late-life depression treatment outcomes [Tunvirachaisakul et al., 2017]) in this population is also needed, as the evidence base for older adults tends to focus on depression to the detriment of other psychiatric disorders (Dham et al., 2017).

\section{Conclusions}

Home-based problem-solving therapy may significantly reduce depressive symptoms in older adults with physical comorbidities affecting functioning in the short term. The evidence for collaborative care is mixed in this population, and effects on functioning, quality of life, and service use are currently unknown. Other therapies and therapies for anxiety lack an evidence base in this population and require further investigation.

\section{Conflict of interest declaration}

RF was funded by the National Institute of Health Research School for Primary Care Research. This paper presents independent research funded by the National Institute for Health Research School for Primary Care Research (NIHR SPCR). The views expressed are those of the author(s) and not 
necessarily those of the NIHR, the NHS or the Department of Health.

The other authors declare no conflicts of interest.

\section{Description of authors' roles}

$\mathrm{RF}$ and $\mathrm{KW}$ designed the review protocol. RF ran the searches, screened references, assessed risk of bias, conducted meta-analysis and drafted the manuscript. YB conducted trials register searches, screened references, assessed risk of bias and provided feedback on manuscript drafts. KW provided feedback throughout the review process, was a third reviewer for resolving any inclusion disagreements and provided feedback on manuscript drafts. All authors have read and approved the final manuscript.

\section{Acknowledgments}

We would like to thank all authors who kindly responded to our requests for further data.

\section{Supplementary material}

To view supplementary material for this article, please visit https://doi.org/10.1017/S1041610218 001564 .

\section{References}

Alexopoulos, G. S., Raue, P. and Areán, P. (2003). Problem-solving therapy versus supportive therapy in geriatric major depression with executive dysfunction. The American fournal of Geriatric Psychiatry, 11, 46-52. doi: 10.1097/00019442$200301000-00007$.

Alexopoulos, G. S. et al. (2016). Clinical case management versus case management with problem-solving therapy in low-income, disabled elders with major depression: a randomized clinical trial. The American fournal of Geriatric Psychiatry, 24, 50-59. doi: 10.1016/j.jagp.2015. 02.007.

Archer, J. et al. (2012). Collaborative care for depression and anxiety problems (Review). Cochrane Database of Systematic Reviews, Art. CD006525. doi: 10.1002/14651858. CD006525.pub2.

Areán, P. A., Raue, P., Mackin, R. S., Kanellopoulos, D., McCulloch, C. and Alexopoulos, G. S. (2010). Problemsolving therapy and supportive therapy in older adults with major depression and executive dysfunction. American Fournal of Psychiatry, 167, 1391-1398. doi: 10.1176/appi. ajp.2010.09091327.

Banerjee, S., Shamash, K., Macdonald, A. J. and Mann, A. H. (1996). Randomised controlled trial of effect of intervention by psychogeriatric team on depression in frail elderly people at home. BMF, 313, 1058-1061. doi: $10.1136 / \mathrm{bmj} .313 .7064 .1058$.

Benraad, C. E. M., Kamerman-Celie, F., van Munster, B. C., Oude Voshaar, R. C., Spijker, J. and Olde Rikkert, M. G. M. (2016). Geriatric characteristics in randomised controlled trials on antidepressant drugs for older adults: a systematic review. International fournal of Geriatric Psychiatry, 31, 990-1003. doi: 10.1002/gps.4443.

Blanchard, M. R., Waterreus, A. and Mann, A. H. (1995). The effect of primary care nurse intervention upon older people screened as depressed. International fournal of Geriatric Psychiatry, 10, 289-298. doi: 10.1002/gps .930100405 .

Braam, A. W. et al. (2014). Depression, subthreshold depression and comorbid anxiety symptoms in older Europeans: results from the EURODEP concerted action. Fournal of Affective Disorders, 155, 266-272. doi: 10.1016/ j.jad.2013.11.011.

Bruce, M. L. et al. (2015). Clinical effectiveness of integrating depression care management into medicare home health: the Depression CAREPATH Randomized trial. FAMA Internal Medicine, 175, 55-64. doi: 10.1001/ jamainternmed.2014.5835.

Bruce, M. L., Lohman, M. C., Greenberg, R. L., Bao, Y. and Raue, P. J. (2016). Integrating depression care management into medicare home health reduces risk of 30- and 60-day hospitalization: the depression care for patients at home cluster-randomized trial. Fournal of the American Geriatrics Society, 64, 2196-2203. doi: 10.1111/jgs .14440 .

Choi, N. G., Nathan Marti, C., Bruce, M. L., Hegel, M. T., Wilson, N. L. and Kunik, M. E. (2014). Six-month postintervention depression and disability outcomes of in-home telehealth problem-solving therapy for depressed, low-income homebound older adults. Depression and Anxiety, 31, 653-661. doi: 10.1002/da.22242.

Ciechanowski, P. et al. (2004). Community-integrated home-based depression treatment in older adults: a randomized controlled trial. Fournal of the American Medical Association, 291, 1569-1577. doi: 10.1001/jama.291 .13.1569.

Cuijpers, P., Li, J., Hofmann, S. G. and Andersson, G. (2010). Self-reported versus clinician-rated symptoms of depression as outcome measures in psychotherapy research on depression: a meta-analysis. Clinical Psychology Review, 30, 768-778. doi: 10.1016/j.cpr.2010.06.001.

Dham, P. et al. (2017). Collaborative care for psychiatric disorders in older adults: a systematic review. The Canadian Fournal of Psychiatry, 62, 761-771. doi: 10.1177/ 0706743717720869.

Djernes, J. K., Gulmann, N. C., Foldager, L., Olesen, F. and Munk-Jørgensen, P. (2011). 13 year follow up of morbidity, mortality and use of health services among elderly depressed patients and general elderly populations. The Australian and New Zealand Fournal of Psychiatry, 45, 654-662. doi: 10.3109/00048674.2011.589368.

El1, K., Unützer, J., Aranda, M., Gibbs, N. E., Lee, P.-J. and Xie, B. (2007). Managing depression in home health care: a randomized clinical trial. Home Health Care Services Quarterly, 26, 81-104. doi: 10.1300/J027v26n03_05.

Enguidanos, S. M., Davis, C. and Katz, L. (2005). Shifting the Paradigm in Geriatric Care Management: 
moving from the medical model to patient-centred care. Social Work in Health Care, 41, 1-16. doi: 10.1300/ J010v41n01_01.

Ferrucci, L. et al. (2004). Designing randomized, controlled trials aimed at preventing or delaying functional decline and disability in frail, older persons: a consensus report. Fournal of the American Geriatrics Society, 52, 625-634. doi: 10.1111/j.1532-5415.2004.52174.x.

Gellis, Z. D., McGinty, J., Horowitz, A., Bruce, M. L. and Misener, E. (2007). Problem-solving therapy for latelife depression in home care: a randomized field trial. American Fournal of Geriatric Psychiatry, 15, 968-978. doi: 10.1097/JGP.0b013e3180cc2bd7.

Gellis, Z. D., McGinty, J., Tierney, L., Jordan, C., Burton, J. and Misener, E. (2008). Randomized controlled trial of problem-solving therapy for minor depression in home care. Research on Social Work Practice, 18, 596-606. doi: 10.1177/1049731507309821.

Gould, R., Coulson, M. and Howard, R. (2012a). Cognitive behavioral therapy for depression in older people: a meta-analysis and meta-regression of randomized controlled trials. Fournal of the American Geriatrics Society, 60, 1817-1830. doi: 10.1111/j.1532-5415.2012 .04166.x.

Gould, R., Coulson, M. and Howard, R. (2012b). Efficacy of cognitive behavioral therapy for anxiety disorders in older people: a meta-analysis and meta-regression of randomized controlled trials. Fournal of the American Geriatrics Society, 60, 218-229. doi: 10.1111/j.1532-5415 $.2011 .03824 . x$.

Gum, A. et al. (2006). Depression treatment preferences in older primary care patients. The Gerontologist, 46, 14-22. doi: 10.1093/geront/46.1.14.

Higgins, J. and Green, S. (eds.) (2011). Cochrane Handbook for Systematic Reviews of Interventions. Oxford: Cochrane Collaboration.

Higgins, J. et al. (2011). The Cochrane Collaboration's tool for assessing risk of bias in randomised trials. BMF, 343, d5928. doi: 10.1136/bmj.d5928.

Hoffmann, T. C. et al. (2014). Better reporting of interventions: template for intervention description and replication (TIDieR) checklist and guide. BMF, 348, 1687. doi: 10.1136/bmj.g1687.

Isaacowitz, D. M. and Seligman, M. E. P. (2002). Cognitive style predictors of affect change in older adults. International fournal of Aging $\mathcal{E}$ Human Development, 54, 233-253. doi: 10.2190/J6E5-NP5K-2UC4-2F8B.

Jonsson, U. et al. (2016). Psychological treatment of depression in people aged 65 years and over: a systematic review of efficacy, safety, and costeffectiveness. PLoS ONE, 11, e0160859. doi: 10.1371/ journal.pone.0160859.

Kiosses, D. N., Arean, P. A., Teri, L. and Alexopoulos, G. S. (2010). Home-delivered problem adaptation therapy (PATH) for depressed, cognitively impaired, disabled elders: a preliminary study. American fournal of Geriatric Psychiatry, 18, 988-998. doi: 10.1097/JGP $.0 \mathrm{~b} 013 \mathrm{e} 3181 \mathrm{~d} 6947 \mathrm{~d}$.

Kiosses, D. N., Ravdin, L. D., Gross, J. J., Raue, P., Kotbi, N. and Alexopoulos, G. S. (2015). Problem adaptation therapy for older adults with major depression and cognitive impairment: a randomized clinical trial.
fAMA Psychiatry, 72, 22-30. doi: 10.1001/jamapsychiatry .2014 .1305 .

Kirkham, J. G., Choi, N. and Seitz, D. P. (2016). Meta-analysis of problem solving therapy for the treatment of major depressive disorder in older adults. International fournal of Geriatric Psychiatry, 31, 526-535. doi: 10.1002/ gps. 4358 .

Kok, R. M., Nolen, W. A. and Heeren, T. J. (2012). Efficacy of treatment in older depressed patients: a systematic review and meta-analysis of double-blind randomized controlled trials with antidepressants. Fournal of Affective Disorders, 141, 103-115. doi: 10.1016/j.jad.2012.02.036.

Lan, X., Xiao, H. and Chen, Y. (2017). Effects of life review interventions on psychosocial outcomes among older adults: a systematic review and meta-analysis. Geriatrics $\mathcal{E}$ Gerontology International, 17, 1344-1357. doi: 10.1111/ggi .12947.

Landreville, P. and Bissonnette, L. (1997). Effects of cognitive bibliotherapy for depressed older adults with a disability. Clinical Gerontologist, 17, 35-55. doi: 10.1300/ J018v17n04_05.

Landreville, P. and Gervais, P. W. (1997). Psychotherapy for depression in older adults with a disability: where do we go from here? Aging $\mathcal{E}$ Mental Health, 1, 197-208. doi: 10.1080/13607869757083.

Lenze, E. et al. (2016). Older adults' perspectives on clinical research: a focus group and survey study. American fournal of Geriatric Psychiatry, 24, 893-902. doi: 10.1016/j.jagp.2016 .07 .022 .

Llewellyn-Jones, R. et al. (1999). Multifaceted shared care intervention for late life depression in residential care: randomised controlled trial. British Medical fournal, 319, 676-682. doi: 10.1136/bmj.319 .7211 .676 .

Meeks, T. W., Vahia, I. V., Lavretsky, H., Kulkarni, G. and Jeste, D. V. (2011). A tune in "a minor" can "b major": a review of epidemiology, illness course, and public health implications of subthreshold depression in older adults. Fournal of Affective Disorders, 129, 126-142. doi: 10.1016/ j.jad.2010.09.015.

Mohlman, J. (2011). A community based survey of older adults' preferences for treatment of anxiety. Psychology and Aging, 27, 1182-1190. doi: 10.1037/a0023126.

Ni Mhaolain, A. M. et al. (2012). Frailty, depression, and anxiety in later life. International Psychogeriatrics, 24, 1265-1274. doi: 10.1017/S10416 10211002110.

Nyunt, M. (2010). Community Screening, Outreach and Primary Care Management of Late Life Depression. Singapore: National University of Singapore.

Orgeta, V., Brede, J. and Livingston, G. (2017). Behavioural activation for depression in older people: systematic review and meta-analysis. The British Fournal of Psychiatry, 211, 1-6. doi: 10.1192/bjp. bp.117.205021.

Panagioti, M. et al. (2016). Association between chronic physical conditions and the effectiveness of collaborative care for depression. $\mathcal{F A M A}$ Psychiatry, 73, 978. doi: 10.1001/jamapsychiatry.2016.1794.

Rodda, J., Walker, Z. and Carter, J. (2011). Depression in older adults. British Medical fournal, 343, d5219. doi: $10.1136 / \mathrm{bmj} . \mathrm{d} 5219$. 
Schuch, F. B. et al. (2016). Exercise for depression in older adults: a meta-analysis of randomized controlled trials adjusting for publication bias. Revista Brasileira de Psiquiatria, 38, 247-254.

Serrano, J. P., Latorre, J. M., Gatz, M. and Montanes, J. (2004). Life review therapy using autobiographical retrieval practice for older adults with depressive symptomatology. Psychology and Aging, 19, 272-277. doi: 10.1037/0882-7974 .19 .2 .272

Simning, A. and Simons, K. V. (2017). Treatment of depression in nursing home residents without significant cognitive impairment: a systematic review. International Psychogeriatrics, 29, 209-226. doi: 10.1017/S10416102 16001733.

Sterne, J. A. C. et al. (2011). Recommendations for examining and interpreting funnel plot asymmetry in meta-analyses of randomised controlled trials. $B M F$, 343, d4002. doi: 10.1136/bmj.d4002.

The Nordic Cochrane Centre, The Cochrane Collaboration (2014). Review Manager Version 5.3 (RevMan).

Tunvirachaisakul, C. et al. (2017). Predictors of treatment outcome in depression in later life: a systematic review and meta-analysis. Fournal of Affective Disorders, 227, 164-182. doi: 10.1016/j.jad.2017.10.008.

Vaughan, L., Corbin, A. L. and Goveas, J. S. (2015). Depression and frailty in later life: a systematic review. Clinical Interventions in Aging, 10, 1947-1958. doi: $10.2147 /$ CIA.S69632.

Von Faber, M. et al. (2016). Older people coping with low mood: a qualitative study. International Psychogeriatrics, 28, 603-612. doi: 10.1017/ S1041610215002264.

Walters, K., Falcaro, M., Freemantle, N., King, M. and Ben-Shlomo, Y. (2018). Sociodemographic inequalities in the management of depression in adults aged 55 and over: an analysis of English primary care data. Psychological Medicine, 48, 1504-1513.

Wang, P. S. et al. (2005). Twelve-month use of mental health services in the United States. Archives of General Psychiatry, 62, 629-640. doi: 10.1001/archpsyc.62.6.629.

Wolitzky-Taylor, K. B., Castriotta, N., Lenze, E. J., Stanley, M. A. and Craske, M. G. (2010). Anxiety disorders in older adults: a comprehensive review. Depression and Anxiety, 27, 190-211. doi: 10.1002/da.20653. 